\title{
A novel biplanar medial opening-wedge high tibial osteotomy: the Z-shaped technique. A case series at 7.2 years follow-up
}

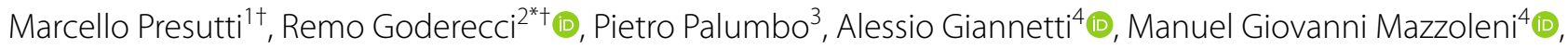
Filippo Maria Nicola Randelli5 ${ }^{5}$, Massimo Angelozzi ${ }^{4}$, Vittorio Calvisi ${ }^{4}$ and Andrea Fidanza ${ }^{4}$

\begin{abstract}
Background: High tibial osteotomy (HTO) provides reliable and good long-term results, if performed with correct indications, but different techniques and types of fixation have been described. The purpose of this study is to present a novel modified biplanar medial opening-wedge (MOW) HTO technique where the osteotomies are performed in a Z-shaped fashion, and to present the medium-term clinical and radiographic results.

Materials and methods: We present a case series of 75 patients (80 knees) with mean age of 45.8 years, affected by isolated medial knee osteoarthritis and symptomatic varus knee malalignment, who underwent novel biplanar Z-shaped MOWHTO. Clinical and radiological outcomes were collected, retrospectively before surgery and at median follow-up of 7.2 years (95\% Cl 5.6-9.2 months) after surgery. Clinical results and satisfaction were assessed by visual analog scale (VAS), Western Ontario and McMaster University Osteoarthritis Index (WOMAC), and Likert scale. Radiological assessment involved the evaluation of the medial proximal tibial angle (MPTA), tibial slope (TS), Caton-Deschamps index, and knee osteoarthritis grade according to Ahlbäck classification. Pre- and postoperative results were compared using the two-tailed $t$-test or Wilcoxon's test of independent samples for paired data or nonparametric analog. $P<0.05$ was considered significant.
\end{abstract}

Results: At medium-term follow-up, Z-shaped MOWHTO showed a survival rate of $95 \pm 1.7 \%$ with failure occurring in four knees due to symptom recurrence and osteoarthritis progression. No perioperative complications were observed (intraarticular fracture, delayed union or nonunion, and neurological injury). Mean bone healing time was 12 weeks. Clinical scores showed significant improvement at last follow-up and a good grade of satisfaction. MPTA increased significantly, while Caton-Deschamps index decreased significantly. No significant TS increase was found.

Conclusions: Modified biplanar Z-shaped MOWHTO is a safe and reliable technique that offers satisfactory clinical and radiological medium-term outcomes with low knee arthroplasty conversion rate. The unique three-dimensional geometrical conformation potentially provides a favorable environment for bone healing, increased anteroposterior and rotational stability, and safer opening-wedge loading force application with low lateral hinge fracture risk.

Level of evidence: Level IV, retrospective observational case series study.

Trial registration The study protocol was approved by the Internal Review Board of our Institution (authorization number 54/2019, 20 November 2019).

\footnotetext{
*Correspondence: remo.goderecci@aslteramo.it

${ }^{+}$Marcello Presutti and Remo Goderecci are co-first authors

${ }^{2}$ Unit of Orthopaedics and Traumatology, "G. Mazzini" Civil Hospital

of Teramo, ASL 4 Teramo, Piazza Italia 1, 64100 Teramo, Italy

Full list of author information is available at the end of the article
}

(c) The Author(s) 2021. Open Access This article is licensed under a Creative Commons Attribution 4.0 International License, which permits use, sharing, adaptation, distribution and reproduction in any medium or format, as long as you give appropriate credit to the original author(s) and the source, provide a link to the Creative Commons licence, and indicate if changes were made. The images or other third party material in this article are included in the article's Creative Commons licence, unless indicated otherwise in a credit line to the material. If material is not included in the article's Creative Commons licence and your intended use is not permitted by statutory regulation or exceeds the permitted use, you will need to obtain permission directly from the copyright holder. To view a copy of this licence, visit http://creativecommons.org/licenses/by/4.0/. 
Keywords: Osteoarthritis, Unicompartmental knee osteoarthritis, High tibial osteotomy, HTO, Medial opening-wedge high tibial osteotomy, MOWHTO, Modified biplanar osteotomy, Z-shaped osteotomy, Puddu plate, Knee

\section{Introduction}

Knee osteoarthritis (OA) is a pervasive orthopedic pathology with significant socioeconomic burden in terms of direct and indirect costs [1].

The most common degenerative pattern involves primarily the medial compartment as a result of an unfavorable load transmission of varus knee deformity [2-5].

The first treatment line is conservative, mainly symptomatic, to delay joint replacement as long as possible [6]. Younger patients with mild OA tend to be dissatisfied after total knee arthroplasty (TKA) [7, 8], and unicompartmental knee arthroplasty (UKA), in case of medial $\mathrm{OA}$ in a varus knee of an active patients, is still discussed but controversial in literature $[9,10]$.

High tibial osteotomy (HTO) is a viable and cost-effective preserving surgery that allows pain reduction and return to physical activities $[11,12]$.

HTO is indicated in younger active but symptomatic patients (generally younger than 65 years old) affected by an arthritic medial compartment, with an axial deformity angle lower than $20^{\circ}$ and a knee flexion range of at least $100^{\circ}$ [13].

Different HTO strategies and types of fixation have been described [14]. Historically, this procedure was commonly performed by lateral closing-wedge HTO (LCWHTO) [15]. This technique still represents an effective treatment but is technically demanding and at higher risk of complications $[13,16]$. LCWHTO is rarely performed nowadays, since the medial opening-wedge HTO (MOWHTO) technique was developed, and became popular, providing several advantages, such as easier and more accurate correction, with good long-term results [17-19]. Other advantages of MOWHTO include: conservation of the proximal tibial anatomy and bone stock which allows easier conversion to TKA; accurate protection of the peroneal nerve, avoiding fibular osteotomy; preservation of the proximal tibiofibular joint; and reduction of compartment syndrome risk [20,21]. Nevertheless, MOWHTO has possible complications including delayed union, posterior slope increase, and patellar height reduction, and higher risk of lateral hinge or cortex fracture $[4,13,22,23]$. MOWHTO was initially described as a single monoplanar osteotomy, oblique or transverse to the frontal plane [24, 25]. Lately, biplanar MOWHTO techniques have been described, such as the V-shaped or retrotubercle osteotomy [26-28]. Both of these techniques are currently those performed most frequently.
The smaller gap volume, and wider bone contact, are expected to promote faster and undisturbed bone ossification [29].

The goal of this study is to present a novel biplanar Z-shaped MOWHTO technique (Fig. 1) and its longterm clinical and radiological outcomes.

This new Z-shaped osteotomy conformation, compared with classic biplanar osteotomies, improves primary stability and firmness by widening the cancellous bone surfaces in contact and orienting one of the three osteotomies on a coronal plane, parallel to the tibial axis.

The hypothesis of the authors was that a biplanar Z-shaped MOWHTO can ensure high survival rates after long-term follow-up, with satisfactory functional and radiological results and low rate of complications.

\section{Materials and methods}

We present a consecutive case series of 75 patients (80 knees) who underwent Z-shaped MOWHTO. All the procedures were performed from 2001 at a single orthopedic center (Casa di Cura Villa Esther in Bojano $(\mathrm{Cb})$, Italy), by a single senior surgeon, who designed the surgical technique. To date, at median follow-up of 7.2 years (95\% CI 5.6-9.2 months), patients have undergone clinical and radiological assessment. All data were collected prospectively and analyzed retrospectively by an independent assessor. All procedures were performed in accordance with ethical standards, and the study protocol was approved by the Internal Review Board of our Institution (authorization number 54/2019, 20 November 2019). All patients were informed and provided written informed consent to participate in the study. The study complied with current national and international laws and regulations governing the use of human subjects (Declaration of Helsinki and its later amendments).

The patients' indications for undergoing Z-shaped MOWHTO were: knee pain associated with varus malalignment combined with isolated medial compartment osteoarthritis of the knee. We considered unsuitable for surgery those patients with active knee flexion lower than $120^{\circ}$ or extension deficiency exceeding $10^{\circ}$. Contraindications for Z-shaped MOWHTO also included high-grade knee ligamentous instabilities, severe knee osteoarthritis (grade 4 and 5 according to Ahlbäck classification), active local or systemic infection, and/or inflammatory arthropathy. The study inclusion criteria were patients who have undergone Z-shaped MOWHTO since January 2001 with available preoperative and postoperative 

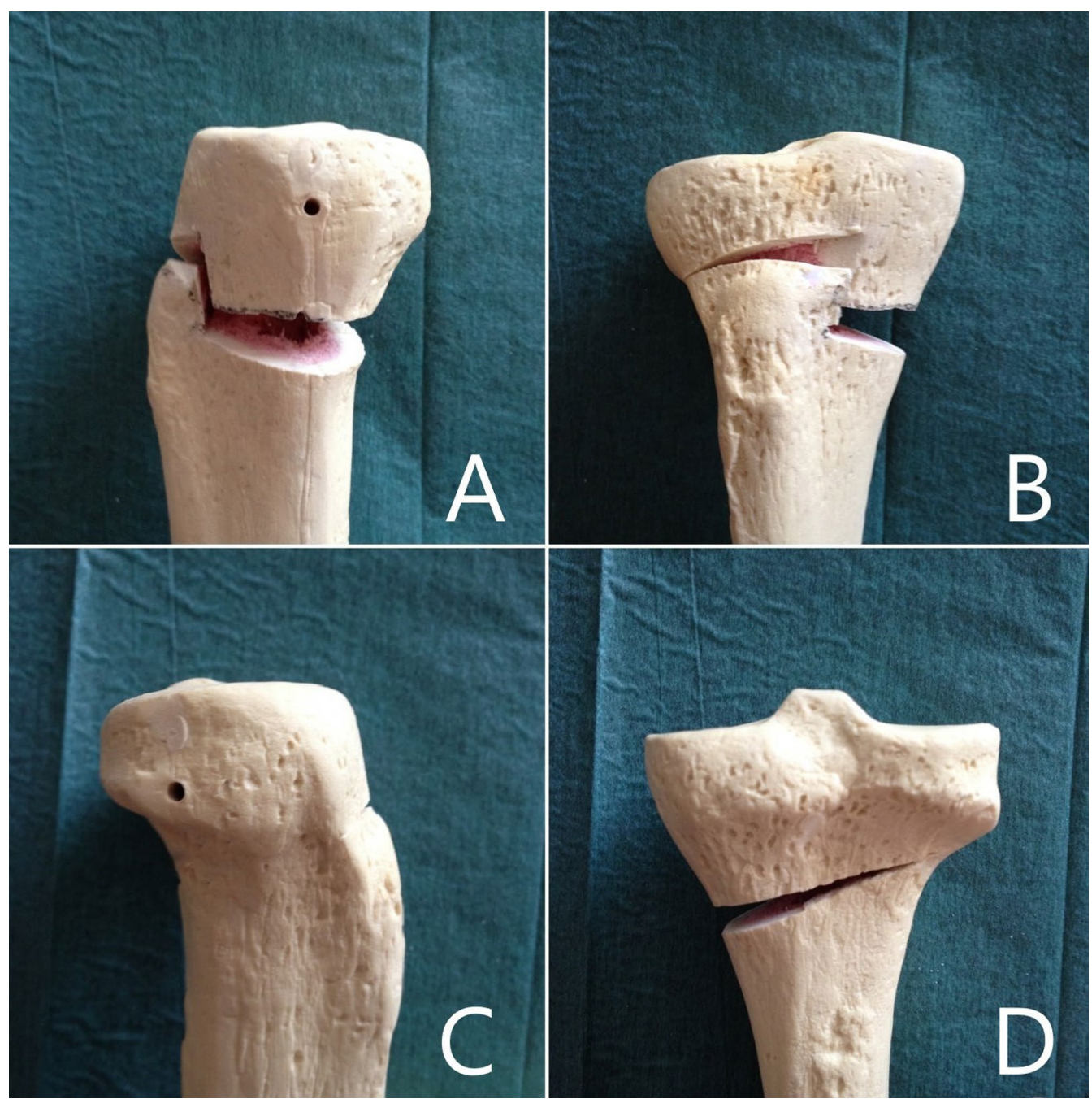

Fig. 1 Reproduction of Z-shaped biplanar medial opening-wedge high tibia osteotomy technique on artificial bone model of left proximal tibial: A medial, $\mathbf{B}$ anterior, $\mathbf{C}$ lateral, and $\mathbf{D}$ posterior view

clinical and radiological data, recorded by the senior surgeon (M.P.) in his personal digital database (VisualMed by Efeso Active Marketing S.r.l). Exclusion criteria from the study were unavailability of preoperative or postoperative clinical or radiological dataset, and unwillingness to participate.

\section{Preoperative assessment}

Preoperatively, the patients were investigated with a full-length standing anteroposterior radiograph of the entire lower extremities for evaluation of alignment of lower limbs. The knee joints were further investigated by bilateral radiographs in Rosenberg view, anteroposterior view, and true lateral view at $30^{\circ}$ knee flexion [30]. The knee osteoarthritis grade was evaluated radiographically according to Ahlbäck classification as follows: grade 1, $30 \%$; grade $2,50.5 \%$; grade $3,19.5 \%$ [31].

\section{Surgical technique}

The surgical procedure is performed with the patient in supine position on a radiolucent operating table and using a pneumatic tourniquet.

The center of the femoral head is identified preoperatively by fluoroscopy and marked with an adhesive metal reference on the inguinal skin. This reference can be palpated through the sterile draping and is used as a landmark for the alignment measurement rod. The center of the ankle and the knee are also determined by fluoroscopy. 
After tourniquet inflation, diagnostic arthroscopy can be performed followed by meniscal and cartilaginous debridement, if indicated, at the medial compartment of the knee. Microfractures or chondroplasty can be performed as needed.

After the first arthroscopic surgical time, the arthroscopic portals are sutured, and the operative field is prepared for the MOWHTO procedure. With the knee flexed at $90^{\circ}$, a $5-8-\mathrm{cm}$ longitudinal skin incision is performed, centered approximately $4 \mathrm{~cm}$ distal to the medial joint line, midway between the posterior medial tibial crest and the tibial tuberosity. After superficial dissection, the underlying sartorial fascia is exposed, incised, and carefully reflected off the underlying gracilis and semitendinosus tendons, which can be identified and detached from their distal insertion. The distal portion of the medial collateral ligament is exposed and elevated from the medial tibial cortex as far as the level of the planned osteotomy. After exposure of the proximal tibia, a blunt Hohmann retractor is placed on the posterior aspect of the tibial metaphysis to elevate and protect the medial collateral ligament and neurovascular structures. This allows exposure of the posteromedial corner of the tibia and facilitates the final placement of the plate on a posteromedial position. A second retractor is placed under the patellar tendon. Under fluoroscopic control, a guide wire is drilled "freehand" from the proximal tibia, approximately $6 \mathrm{~cm}$ distal from the joint line, in a medial to lateral and upward direction (Fig. 2). The guide wire is directed approximately $20^{\circ}$ upwards, aiming the superior part of the head of the fibula. Once the correct orientation of the first guide wire is obtained, under fluoroscopic guidance, two additional wires are drilled parallel to the first one, with the assistance of a first specific self-made guide (Fig. 2). The drilled wires are measured to calculate the exact length of the osteotomy.

Using a special self-made osteotomy cutting guide (Fig. 3), inserted on the three guide wires, the first, 2-cm vertical osteotomy is performed on a coronal plane, posteriorly to the tibial tuberosity, through an oscillating bone saw and completed with a sharp thin osteotome without violating the lateral cortex (Fig. 4). The second, $1-\mathrm{cm}$ horizontal osteotomy is performed on a oblique plane just above the tibial tuberosity behind the patellar tendon, oriented at approximately $120^{\circ}$ with respect to and starting at the proximal end of the first coronal osteotomy (Fig. 5). This second osteotomy is completed, including the lateral cortex of the tibial tubercle, using a thin 1-cm large osteotome.

The third final horizontal osteotomy involving $70 \%$ of the tibial width starts from the posterior aspect of the tibia. The osteotomy is performed in posteromedialanterolateral direction, just below the three guide wires, using an oscillating saw and carefully completed with a thin sharp 2-cm large osteotome. The oblique upward direction plane of this last osteotomy is parallel and in

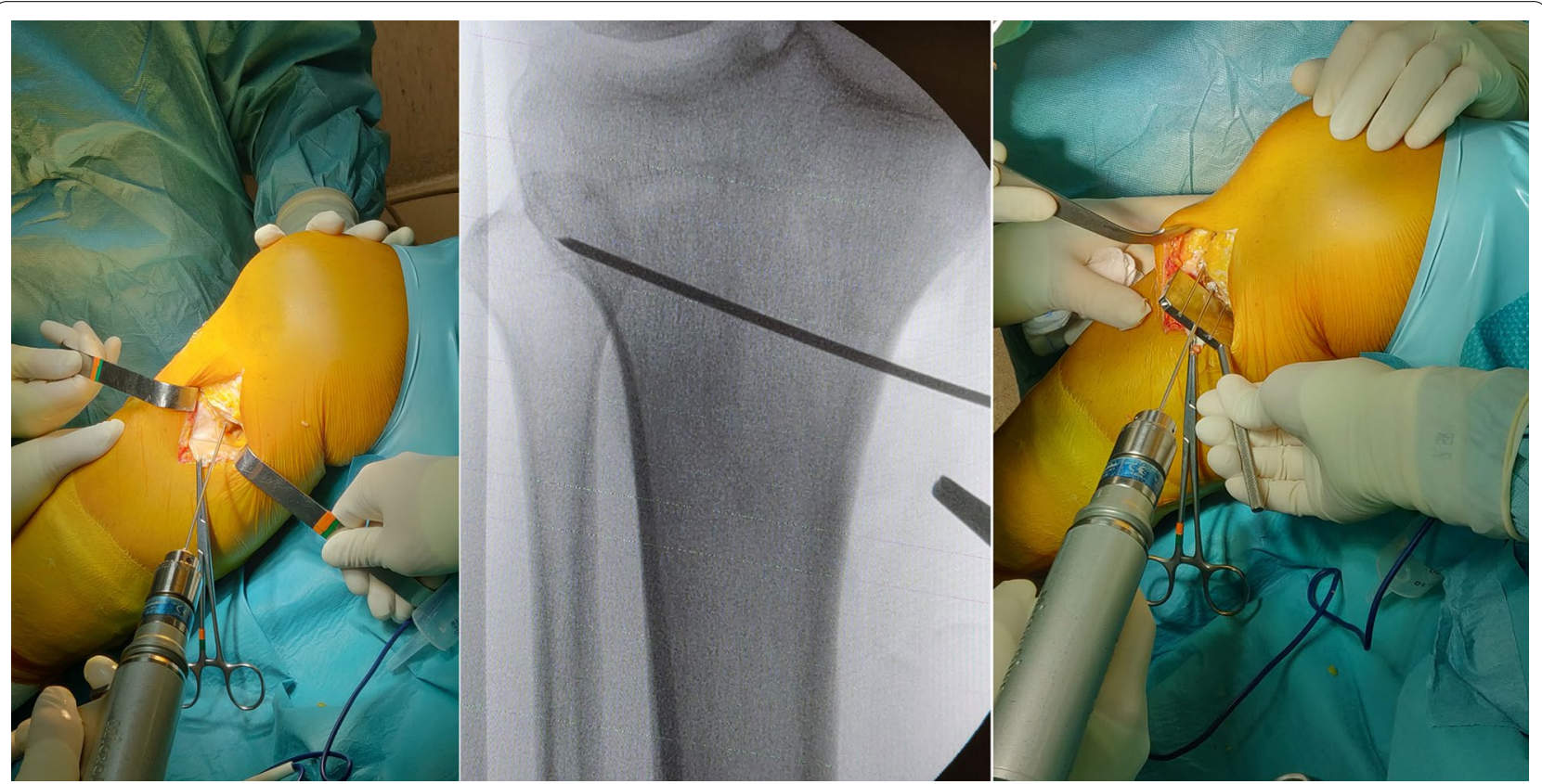

Fig. 2 Intraoperative findings and fluoroscopic imaging of the first guide wire drilled through the proximal right tibia from medial to lateral directed $20^{\circ}$ upwards. The first wire is advanced, under fluoroscopic guidance, toward the superior part of the fibular head. On the right is shown the positioning of the two additional parallel wires with the assistance of a self-made guiding device 


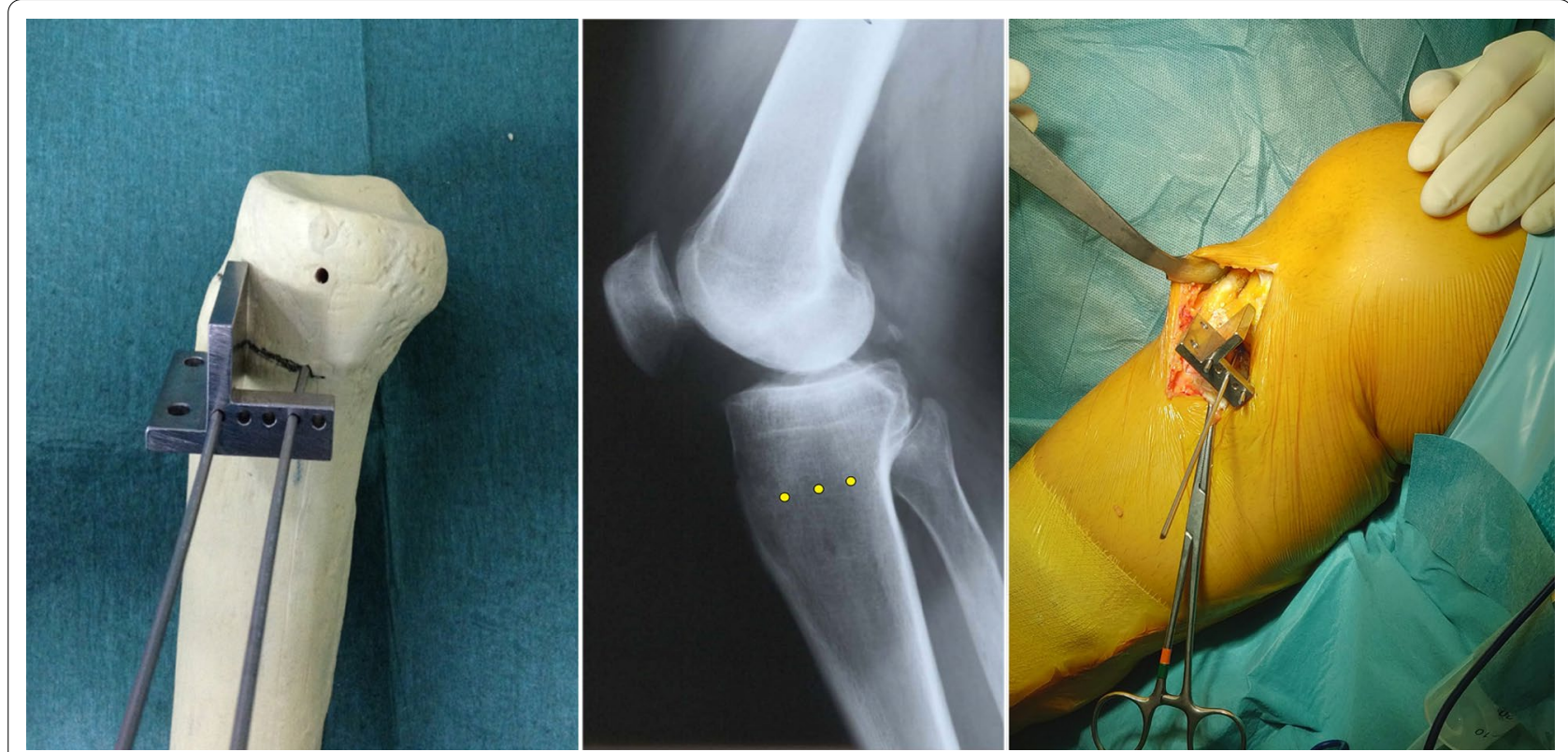

Fig. 3 On the left, a demonstration of the second self-made cutting guide on a right tibia bone model. In the middle, a graphical representation of the three guide wires (yellow dots) on a lateral X-ray view of a right knee. On the right, the intraoperative findings of the self-made cutting guide positioning over the three wires, in a right tibia

strict contact with the three guide wires to prevent its accidental proximal migration into the joint. The osteotomy is completed without affecting the lateral cortex and preserving the anterior cortex and tibial tubercle.

The complete osteotomy should appear Z-shaped in a sagittal plane, making certain that all the cancellous metaphysis and, especially, only the posterior cortex is completely interrupted, preserving a lateral hinge of about $1 \mathrm{~cm}$ of intact bone (Fig. 6).

Then, a distractor is inserted into the major oblique osteotomy site to gradually open the gap until adequate correction is achieved. The correct alignment restoration is verified under fluoroscopy, through a guide rod, placed from the center of the femoral head to the center of the talus. On the image intensifier centered on the knee, the guide rod should lie just lateral to lateral tibial spine, leaving $62.5 \%$ of the tibial plate width medially (Fig. 7). Alignment correction is possible by opening or closing the gap according to the preoperative planning and under direct fluoroscopic control. The fixation of the osteotomy is achieved using the Tibia Opening Wedge Osteotomy Plate (Arthrex Inc., Naples, FL, USA), also known as the "Puddu plate." The plate is positioned on the medial cortex of the tibia as posteriorly as possible and fixed proximally with two cancellous screws and distally with two cortical screws (Fig. 8). The osteotomy gap is not filled with bone graft or bone substitute; only a local bridge of free autologous cancellous bone, between the proximal and the distal side of the osteotomy, is developed using a standard curette in the wider medial portion of the gap to promote healing (Fig. 8). Final fluoroscopic assessment ensures adequate positioning of the hardware. After the implant of the Puddu plate the pes anserinus is reinserted, suction drain is placed, and the wound is closed in layered fashion.

\section{Postoperative aftercare}

Antithrombotic prophylaxis (low-molecular-weight heparin) was administered for 40 days in all patients, starting from the first postoperative day. Drains were removed on the second postoperative day, after which walking with two crutches without weight-bearing was allowed. The rehabilitation program included a long-leg hinged brace for 4 weeks. Limited continuous passive motion (CPM; $0-40^{\circ}$ ) was begun on the first postoperative day, and $90^{\circ}$ knee flexion was achieved on the third postoperative day, with the assistance of a physiotherapist. Partial weightbearing began 40 days after surgery, and full weight-bearing after 60 days.

\section{Clinical assessment}

Patients were evaluated preoperatively then at last followup by the same surgeon who performed the procedure.

All measurements and complications (intraoperative fracture, persistent pain, delayed union or nonunion, infection, and thromboembolism) were recorded. 


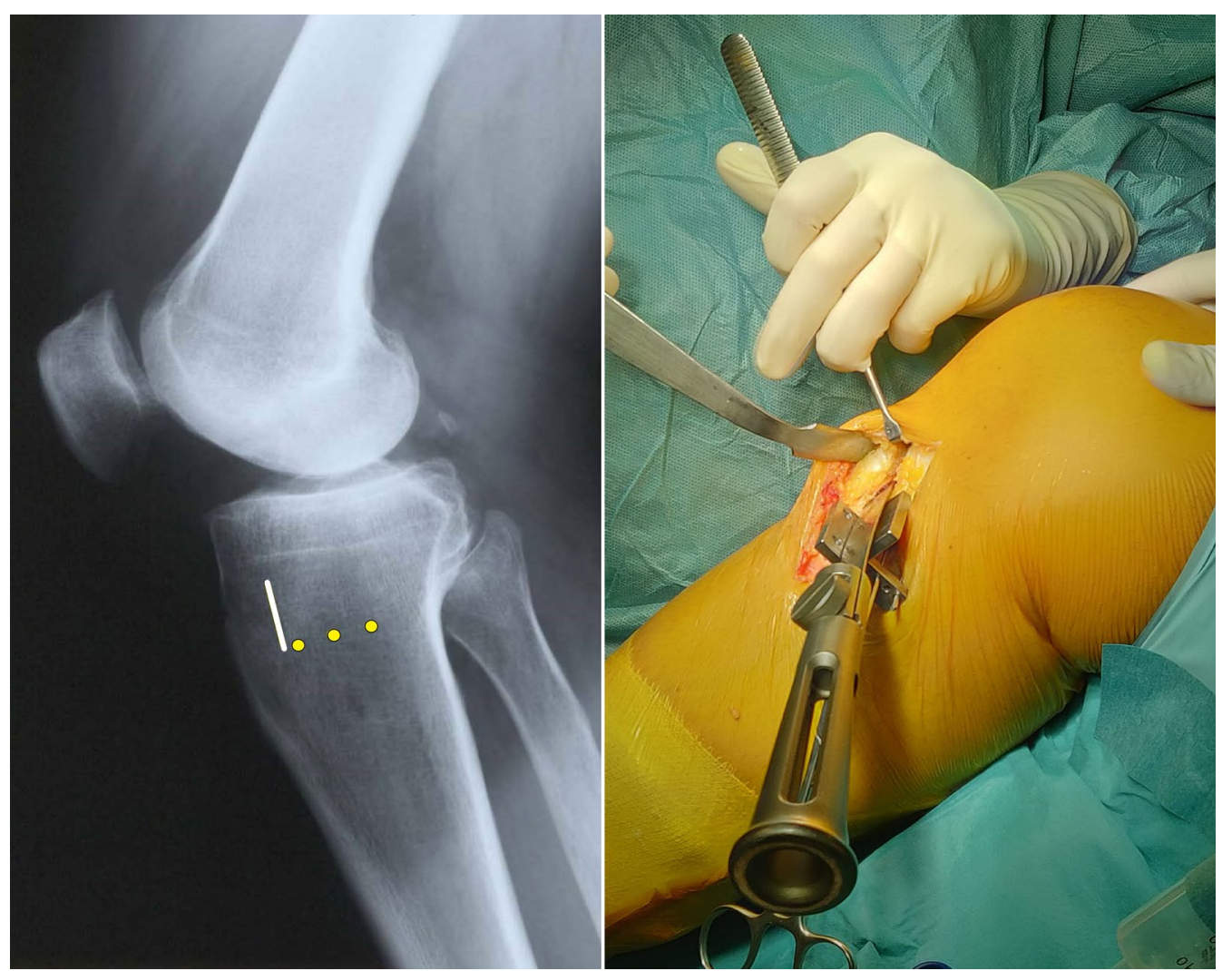

Fig. 4 On the left, a graphical representation of the three guide wires (yellow dots) and the first coronal osteotomy (vertical white line), on a lateral X-ray view of a right knee. On the right, the intraoperative findings of the first coronal osteotomy completed with a sharp thin 2-cm large osteotome, in a right tibia, with the assistance of the self-made cutting guide

Clinical evaluation of knee pain was measured using a self-administered $100-\mathrm{mm}$ visual analog scale (VAS) (0 mm, no pain; $100 \mathrm{~mm}$, worst pain) [32]. Pain was evaluated upon walking. Functional outcomes were assessed by WOMAC [33].

At last follow-up, patients were asked about their satisfaction with the surgery in general. The responses were graded on a five-point Likert scale from "totally satisfied" to "very dissatisfied" [34]. All the clinical data were recorded by the senior surgeon (M.P.) in his personal digital database (VisualMed by Efeso Active Marketing S.r.l).

\section{Radiographic assessment}

Standard anteroposterior and lateral view radiographs of the affected knee were performed on the first day postoperatively then at $1,3,6$, and 12 months postoperatively. At last follow-up, all the patients also underwent a fulllength standing anteroposterior radiograph of the entire lower extremities. Radiographic analysis was performed by the same independent assessor (orthopedic surgeon) to determine the following: tibiofemoral osteoarthritis grade in the Ahlbäck classification system [31], tibial slope (TS) using the angle between the joint line and the perpendicular to the posterior tibial cortex line [35], Caton-Deschamps index (CD) of patellar height [36], and degree of bone healing.

Correction in the frontal plane was evaluated by comparing the medial proximal tibial angle (MPTA), preoperatively and on the follow-up radiographs [37]. MPTA was defined as the angle between the proximal anatomical axis of the tibia and a tangent along the articular surface of the tibial plateau and can be used to predict the varus degree correction [38].

\section{Statistical analyses}

Statistical analysis was carried out using SPSS for Windows 16.0 (SPSS, Inc., Chicago, IL). Shapiro-Wilk test was used to evaluate the normality of distributions of variables. Paired data (preoperative versus postoperative values) that passed the Shapiro-Wilk test for normality were compared using Student's $t$-test, while nonparametric variables were analyzed using Wilcoxon's test. The Kaplan-Meier method was applied to assess data that 


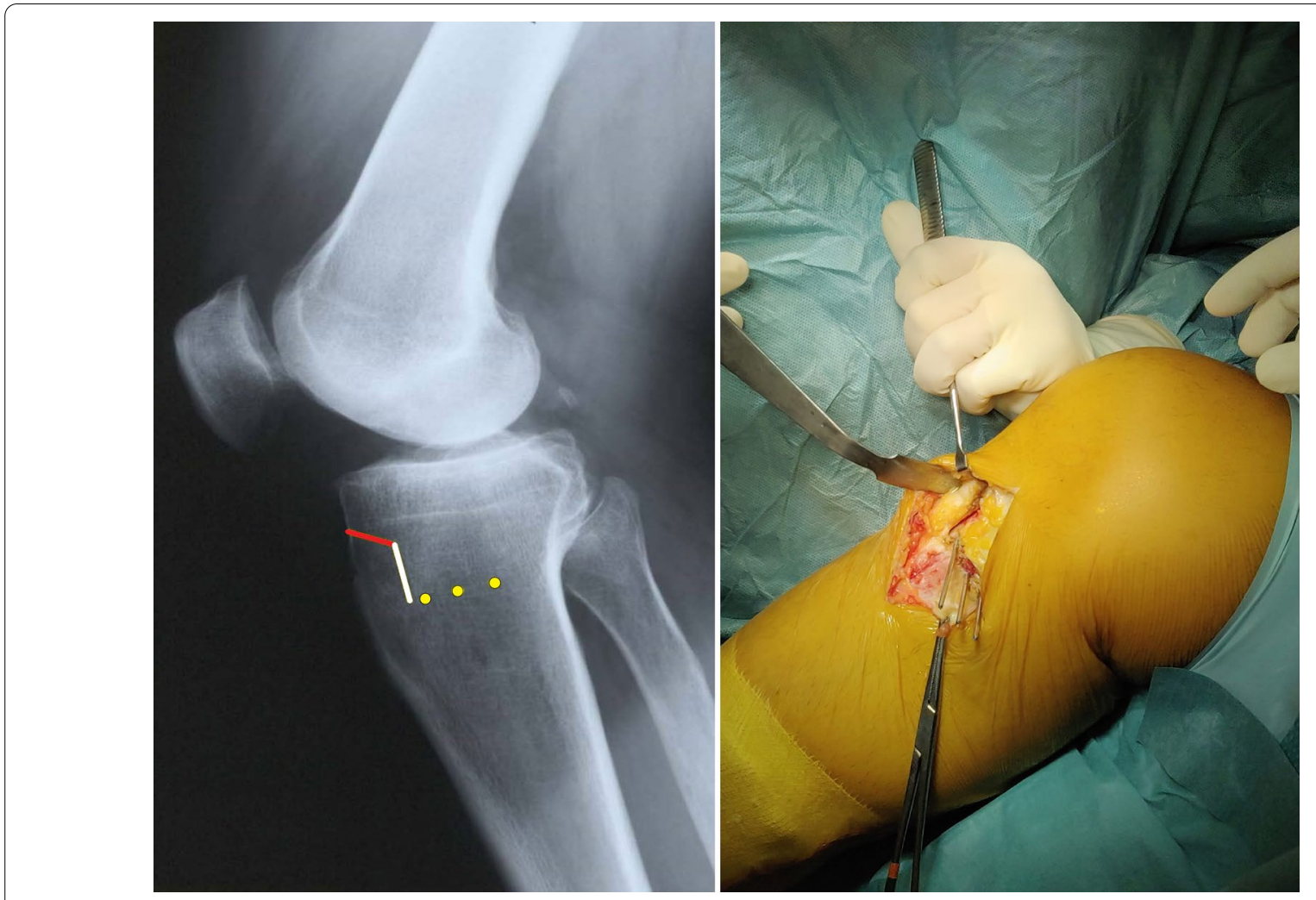

Fig. 5 On the left, a graphical representation of the three guide wires (yellow dots), the first coronal osteotomy (vertical white line) and the second osteotomy (red line) on an oblique plane, on a lateral X-ray view of a right knee. On the right, the intraoperative findings of the first two osteotomies, in a right tibia. The three wires are still positioned to guide the next last osteotomy
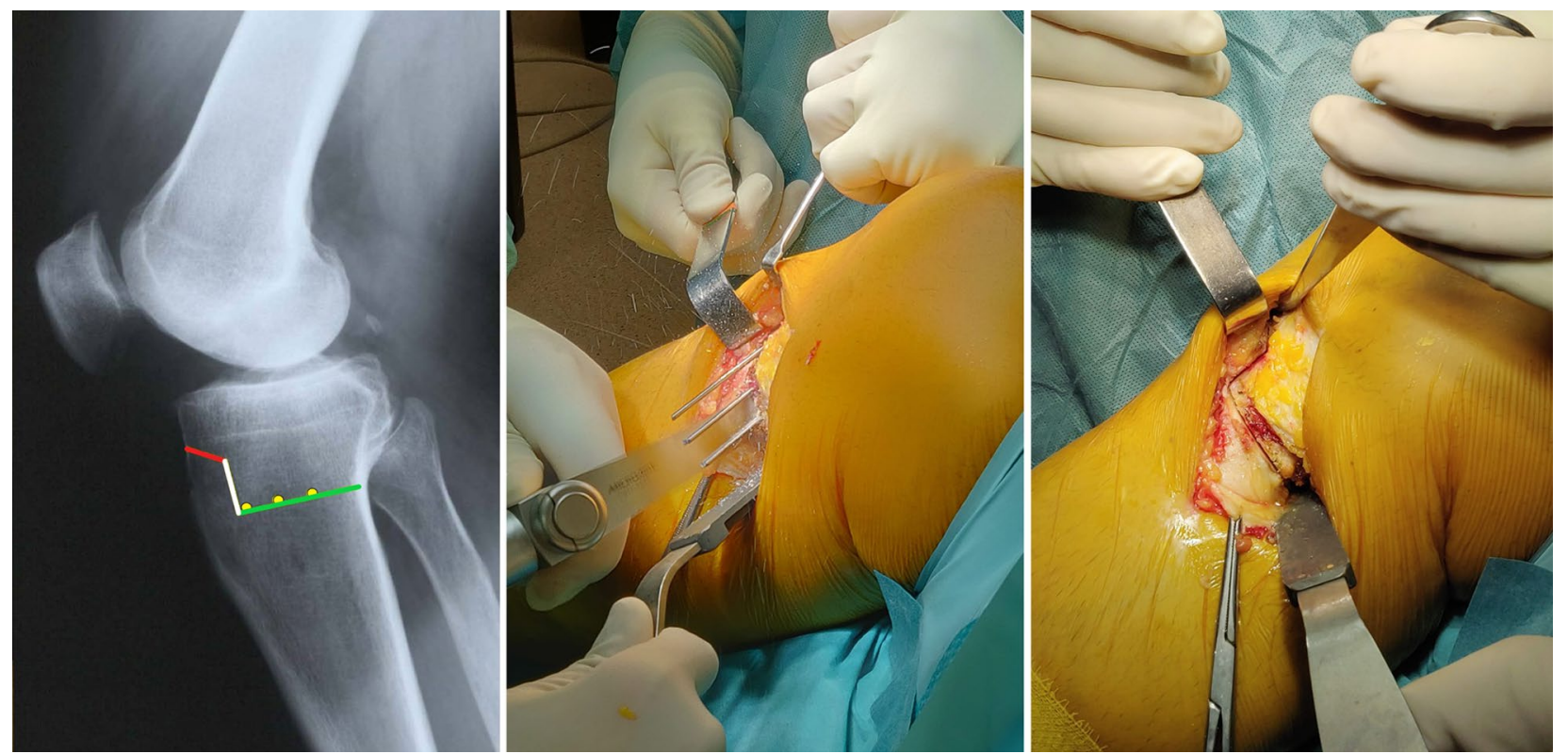

Fig. 6 On the left, a graphical representation of the three guide wires (yellow dots), the first coronal osteotomy (vertical white line), the second oblique osteotomy (red line), and the third horizontal main osteotomy, on a lateral X-ray view of a right knee. In the middle, intraoperative findings of the third horizontal osteotomy performed with an oscillating saw. On the right, the intraoperative findings of complete Z-shaped MOWHTO, in a right tibia, from medial view 


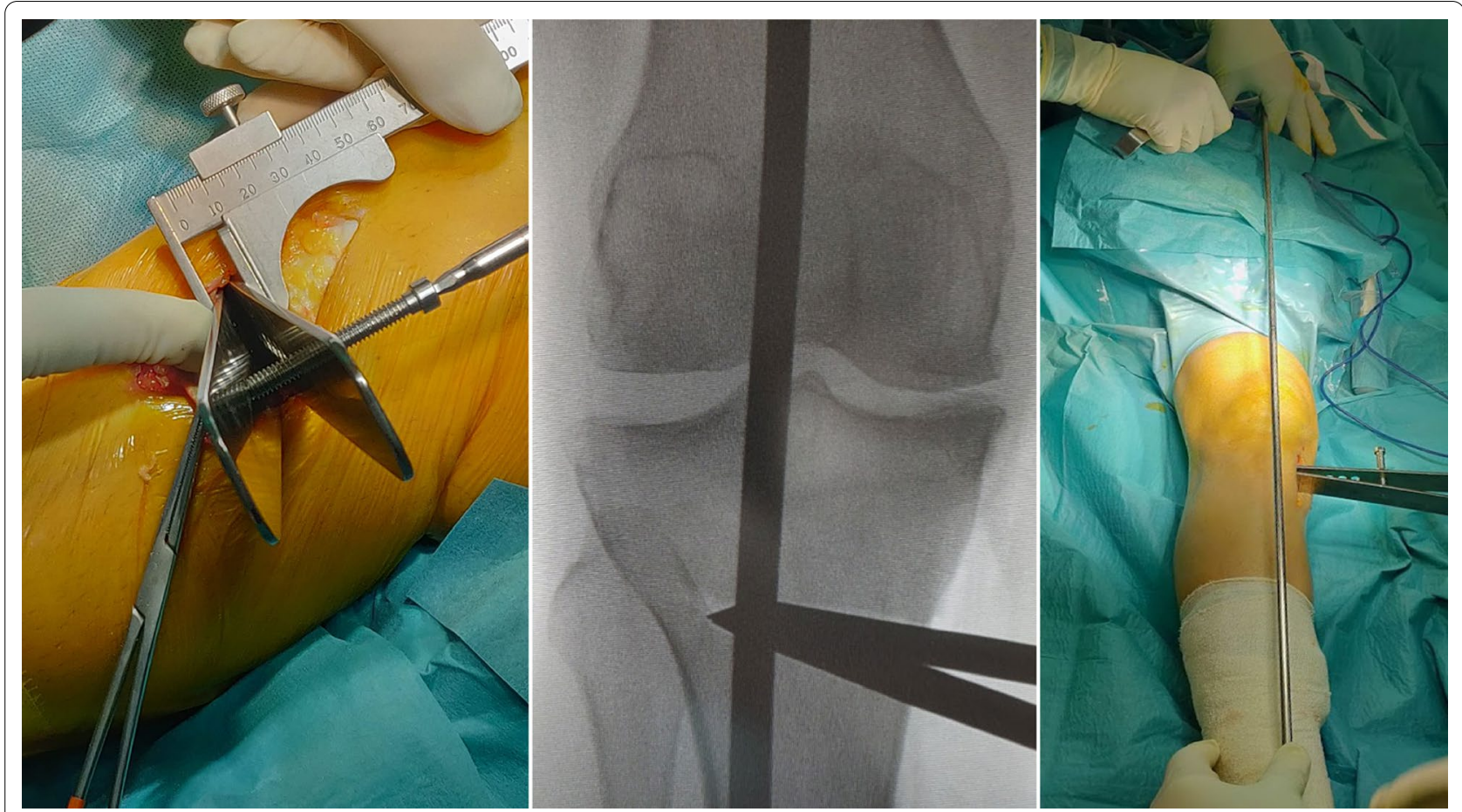

Fig. 7 On the left, intraoperative findings of the distractor positioned and gradually opened into the major horizontal osteotomy site, on a right tibia from medial view. In the middle, the intraoperative alignment fluoroscopic control, verified through the rod guide of a right knee. On the right, the intraoperative finding of the varus correction of the right lower limb, through the distractor and verified through the rod guide

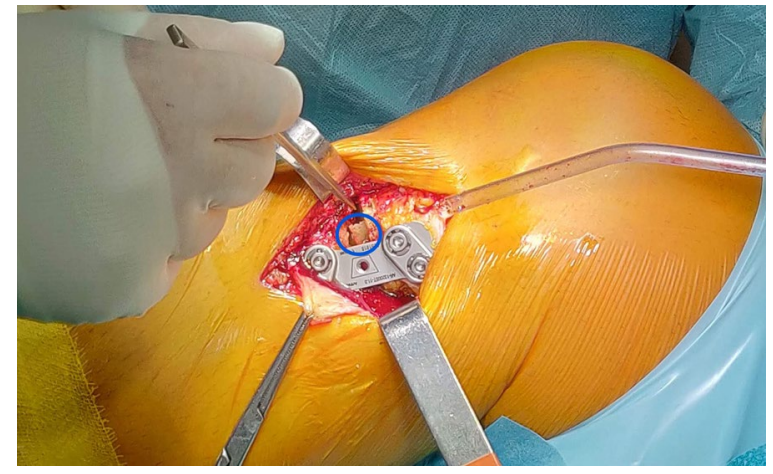

Fig. 8 Intraoperative finding of the Puddu plate positioned and fixed on the posteomedial aspect of the main horizontal osteotomy site, from medial view. The cancellous bone bract detached from both the proximal and distal sides of the site of osteotomy to create a bridge of bone contact that favors healing of the fracture can be seen in the circle

were considered censored, namely survival and revision surgery. $P<0.05$ was considered significant.

\section{Results}

\section{Enrollment process}

The principal independent assessor (P.P.) identified and enrolled, according to the eligibility criteria, 75 patients
(80 knees) who underwent Z-shaped MOWHTO from January 2001. All the data were retrieved from the senior surgeon's (M.P.) personal digital database (VisualMed by Efeso Active Marketing S.r.l). Data were not available for five $(6.7 \%)$ patients who were considered lost at final follow-up and one patient (1.3\%) who died (Table 1).

\section{General results and demographic analysis}

The study included 80 knees of 75 patients, 54 male and 21 female, with mean age of 45.8 years (range 29-62 years). Mean preoperative body mass index (BMI) was $27.3 \pm 3.4 \mathrm{~kg} / \mathrm{m}^{2}$ (Table 2).

After median follow-up of 7.2 (95\% CI 5.6-9.2) years, 74 knees in 69 patients were assessed based on an in-person visit and imaging studies.

Z-shaped MOWHTO failed in four knees, after a mean of $9.25 \pm 1.86$ years. In all four cases, total knee arthroplasty was performed because of recurrence of symptoms and radiological progression of osteoarthritis (MPTA values at revision surgery: $84.5^{\circ}, 83.6^{\circ}, 85.2^{\circ}$, and $82.8^{\circ}$, respectively).

Kaplan-Meier analysis showed an overall survival rate at 10 years of $95 \pm 1.7 \%$. Only 33 knees $(44,6 \%)$ were assessed beyond the median follow-up time of this study, at 8 years after surgery, showing a survival rate of $100 \%$ (Fig. 9). 
Table 1 Enrollment process flow diagram

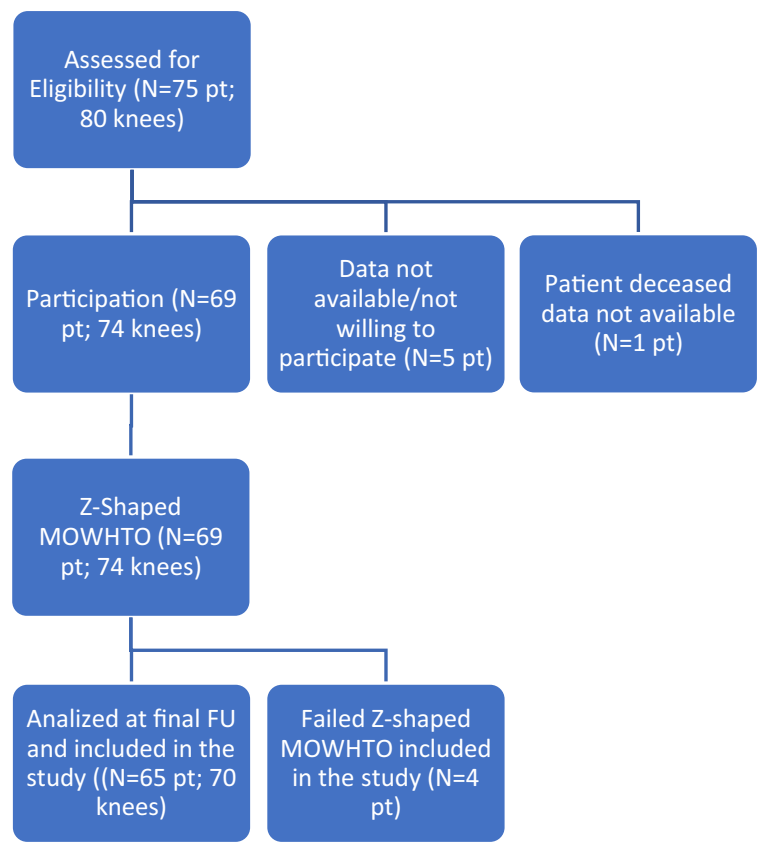

Table 2 Demographic analysis at baseline

\begin{tabular}{ll}
\hline Patient characteristics ( $\boldsymbol{N}=\mathbf{7 5}$ patients, 8 knees) & \\
\hline Gender & \\
Male (\%) & $54(72 \%)$ \\
Female (\%) & $21(28 \%)$ \\
Mean (range) age (years) & $45.8(29-62)$ \\
Mean \pm SD BMl (kg/m ${ }^{2}$ ) & $27.3 \pm 3.4$ \\
\hline
\end{tabular}

Patients' demographic data before surgery. The study included, at baseline, 75 patients for a total of 80 knees. Gender is reported in absolute values and percentage

\section{Complications}

No patients were affected by any of the following complications: lateral tibial cortex or intraarticular fracture, deep venous thrombosis, pulmonary emboli, superficial or deep infection, neurovascular injuries, delayed union or nonunion, or delay in rehabilitation program. One plate and screw rupture was observed before removal.

\section{Clinical outcomes}

The detailed results of the clinical scores (VAS, WOMAC) are presented in Table 3. Statistically significant improvements $(P<0.001)$ of both scores compared with preoperatively were observed at final follow-up.

Most of the patients were satisfied ("totally satisfied" $58 \%$, "fairly satisfied" $26 \%$, or "slightly satisfied" $13 \%$ ) with the surgery at last follow-up, while only two patients were "slightly dissatisfied" (3\%).

\section{Radiological outcomes}

The mean MPTA changed significantly from $83.8 \pm 1.2^{\circ}$ preoperatively (range $81.6-86.5^{\circ}$ ) to $90.5 \pm 1.1^{\circ}$ at last follow-up (range $\left.89-93.5^{\circ}\right)(P<0.001)$. The mean correction angle was $6.7 \pm 1^{\circ}$.

The TS increased, by a mean of $0.4^{\circ}(P=0.187)$. The Caton-Deschamps index decreased significantly, by a mean of $0.14(P<0.001)$ (Table 3$)$.

Mean time to bone healing was 12 weeks, defined as full osteotomy gap filled with new bone (Fig. 10). No relationship was found between the difference in MPTA compared with change in tibial slope, BMI, or age.

\section{Discussion}

This retrospective series of 74 knees treated with MOWHTO using a novel Z-shaped osteotomy showed good results for both clinical and radiological outcomes at 7.2 years follow-up.

These results are similar to outcomes of biplanar osteotomy previously reported [4-6]. Overall, the MPTA was slightly overcorrected, compared with the normal range $\left(85-90^{\circ}\right)$ [18], although the degree of overcorrection achieved in these patients is considered clinically irrelevant [39].

Tibial slope did not increase significantly. The posterior tibial slope increase can be prevented with a simple distraction at the most posterior aspect of the osteotomy gap and with the subsequent positioning and fixation of the plate slightly posterior to the tibial axis [21, 24, 25]. Equal anterior and posterior medial gaps might increase the tibial slope because of the anatomic characteristics of the proximal tibia $[19,40]$.

Conversely, the Caton-Deschamps index decreased significantly postoperatively, but as highlighted in a recent review by Mingliang et al., this change does not affect short-term patient satisfaction [13].

In terms of clinical evaluation, significant improvements in the VAS and WOMAC scores were observed. The positive correlation between the preoperative and final VAS and WOMAC scores shows that clinical scores improved when pain decreased.

In this study, we report only one case of delayed healing, in a heavy smoker. Smoking and overweight/obesity play a major role in development of nonunion in patients treated with HTO [41].

No fracture of the lateral cortex was reported. The incidence of fractures in MOWHTO is significantly higher than in lateral closing-wedge HTO [13]. This complication may lead to instability at the osteotomy site, delayed union, nonunion, and recurrent varus [26]. A meticulous surgical procedure is a critical step to decrease the incidence of lateral cortical hinge fracture. Lateral cortical 


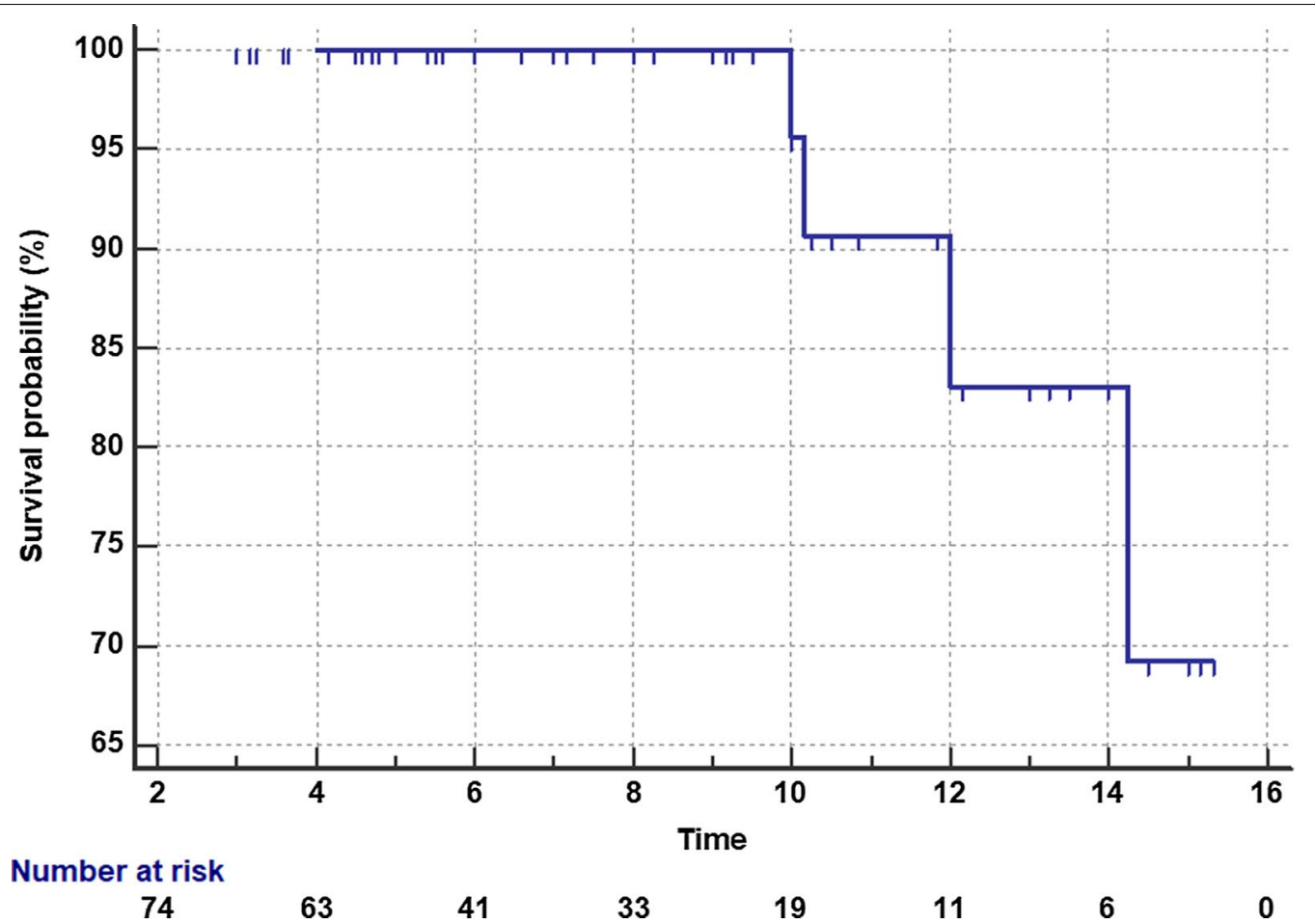

Fig. 9 Kaplan-Meier (K-M) survival plot. The K-M estimator analysis showed an overall survival rate at 10 years of $95 \pm 1.7 \%$

fracture during MOWHTO has been significantly associated with wedge size and opening distance [42, 43]. The risk of lateral cortex fracture is correlated with osteotomy angle amplitude, even reaching $90 \%$ when the correction angle is higher than $8^{\circ}$ [44-46]. The biplanar biomechanical properties of MOWHTO compared with uniplanar MOWHTO allow the application of higher openingwedge load and the achievement of larger correction degrees before encountering lateral cortex fracture [42]. Therefore, because of its effective reduction of the incidence of lateral cortical fracture, biplanar MOWHTO should be preferred over uniplanar MOWHTO [13, 47].

Furthermore, biplanar MOWHTO produces smaller volumes of "empty gaps" combined with larger cancellous

Table 3 Clinical and radiographic outcomes

\begin{tabular}{llll}
\hline & Preoperative & Last follow-up & $P$ value \\
\hline VAS & $5.32 \pm 0.7$ & $0.9 \pm 0.9$ & $<0.001$ \\
WOMAC & $50.1 \pm 11.9$ & $12.7 \pm 8.1$ & $<0.001$ \\
MPTA & $83.8 \pm 1.2^{\circ}$ & $90.5 \pm 1.1^{\circ}$ & $<0.001$ \\
TS & $4.1 \pm 2.9^{\circ}$ & $4.5 \pm 3.1^{\circ}$ & 0.187 \\
CD index & $0.90 \pm 0.14$ & $0.76 \pm 0.11$ & $<0.001$ \\
\hline
\end{tabular}

VAS visual analog scale, WOMAC Western Ontario McMaster University Osteoarthritis Index, MPTA medial proximal tibial angle, TS tibial slope, $C D$ Caton-Deschamps bone surface in contact, compared with the uniplanar MOWHTO technique [47]. Literature describes two healing processes occurring simultaneously after MOWHTO: primary bone healing between the bone surfaces in contact, and secondary bone healing within the gap [13, $47,48]$. Since contact healing is faster than gap healing under stable conditions, the geometrical conformation of biplanar MOWHTO theoretically promotes osteotomy healing, allowing wider bone contact surfaces [47]. This could also explain the good efficacy of rapid bone healing in our series with the exclusive use of local bone autograft without the need to fill the gap with bone harvested from iliac crest or synthetic substitutes [49]. Goshima et al. report that, in around $60 \%$ of the "V-shaped" biplanar MOWHTOs performed in their study, only $25 \%$ of the volume of the osteotomy gap was filled 3 months postoperatively [50]. In contrast, Staubli et al. [51] reported that, on standard radiographs, at least $75 \%$ of the gap was filled with new bone within 6-18 months.

In this study, the average osteotomy healing time was 3 months. We thus deduce that Z-shaped biplanar, rather than V-shaped biplanar MOWHTO, better reflects the geometrical principles required for bone healing, providing an even larger cancellous bone surface combined with a smaller wedge gap volume. Thus, this newly described open-wedge Z-shaped osteotomy may allow the creation of a larger gap, maintaining larger bone surface contact 


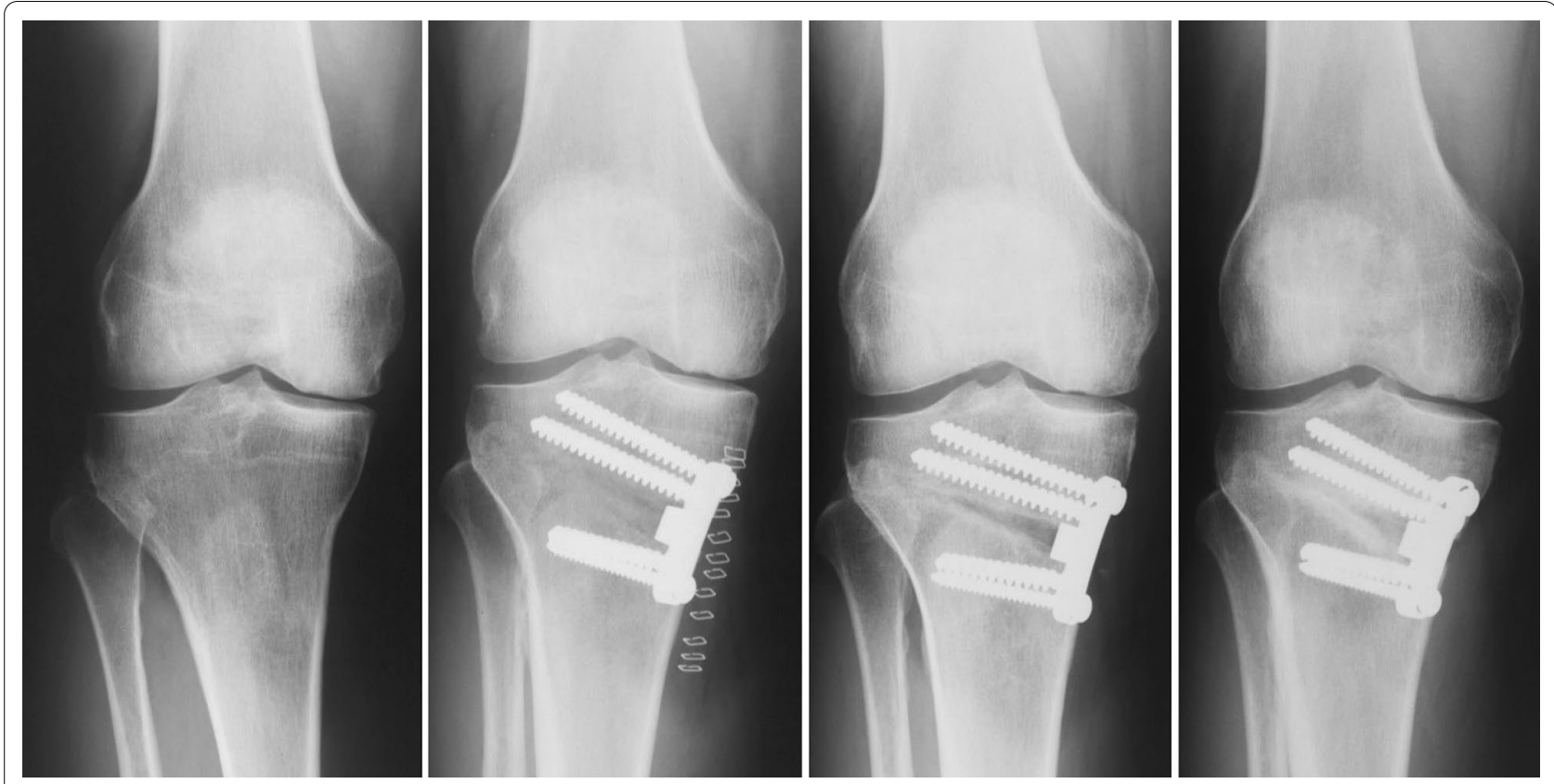

Fig. 10 Anteroposterior $X$-ray views of the same right knee. From left to right, respectively: preoperatively, and 1 day, 1 month, and 3 months postoperatively

areas, compared with the "classic," V-shaped biplanar technique and uniplanar techniques.

Pape et al. demonstrated in a cadaveric study that biplanar osteotomy significantly increased the fixation stability in anteroposterior and rotational planes, especially when short spacer plates, such as the Puddu plate, are used [52]. The additional biomechanical stabilizing effect of V-shaped biplanar MOWHTO and the biologic bone healing improvement reported are both potentially enhanced by the geometric structure of the Z-shaped biplanar MOWHTO presented.

The long-term survivorship is higher after MOWHTO than lateral-closing wedge HTO (LCWHTO), with lower fracture rate [13]. In a recent systematic review, Mingliang et al. reported a pooled 10-year survival rate that was 6.2\% greater for MOWHTO than LCWHTO [13]. In this study, the survival rate at median follow-up of 7.2 years after Z-shaped MOWHTO was $95 \pm 1.7 \%$, in line with the survival rate presented in literature for MOWHTO (91.6\%) [13].

Currently, the most common surgical interventions performed for the treatment of medial osteoarthritis (OA) of the knee are MOWHTO and medial unicompartmental knee arthroplasty (UKA). To the best of the authors' knowledge, a wide literature suggests that good outcomes can be achieved after either procedure. A recent systematic review investigating the return to physical activity after HTO or UKA of 2023 pooled patients showed equal or improved scores, for activity and knee function, regardless of the operation performed [53]. Traditional indications for HTO, such as younger age and $\mathrm{BMI}<30 \mathrm{~kg} / \mathrm{m}^{2}$, were confirmed in that systematic review [53]. The authors reported that the HTO group was 12.2 years younger than the UKA group, with mean age at surgery of 48.4 and 60.6 years, for the HTO and UKA group, respectively. Their findings are in line with the mean baseline age of the patients in the present study (45.8 years) [53]. A Finnish registry-based study on survivorship after 3195 HTOs clearly showed that patients aged less than 50 years had significantly lower risk of conversion to TKR than older people [54]. This allows us to speculate that, despite the similar clinical results of the two procedures, if patients undergo UKA at younger age than indicated, attention must be paid to the subsequent risk of revision to total knee arthroplasty (TKA) [53]. A previous metaanalysis reported a mean revision time to TKA, after UKA, of 8.2 years after surgery, but 9.7 years after HTO [55]. Furthermore, considering that TKA after UKA showed an increased risk of undergoing "re-revision," when compared with TKA after HTO, we emphasize that caution should be taken when UKA is offered to patients younger than 60 years old [56].

There are several limitations to this study.

The first limitation is reproducibility. The self-made guides allow the performance of the osteotomies safely, precisely, and easily, but these devices are essential to 
this technique. A standardized multicentric study should further examine the surgical learning curve and better evaluate intraoperative complications. Although data were recorded prospectively, this is a retrospective outcome evaluation of surgeries performed by a single senior surgeon. The loss of MPTA angle correction over time (difference between the early postoperative value and the value at last follow-up) was not assessed. Another limitation consists in the wide variability of age among the patients. This may have led to some bias in the results presented.

Furthermore, since many heterogeneous methods have been described to assess osteotomy healing on radiographs, and since X-rays do not usually even provide an adequate representation of the osteotomy gap and thus ossification, we decided to define the osteotomy as healed only when we could see the gap completely filled. This methodological flaw made it difficult to compare the gap filling timing with results reported in literature.

Finally, even if this study benefits from clinical and radiographic evaluation at 7.2 years of follow-up, performed by an independent assessor, MOWHTO outcomes could deteriorate over time, particularly after 10-15 years, requiring longer follow-up to provide a definitive conclusion on this technique $[18,57]$.

\section{Conclusions}

With a survival rate of over $95 \%$ at 7.2 years follow-up, MWHTO with a Z-shaped technique seems to be a reliable treatment option for managing medial knee compartment osteoarthritis with varus malalignment in younger active patients. Modified biplanar Z-shaped MOWHTO is a safe and reliable technique that offers satisfactory clinical and radiological medium-term outcomes. The distinctive three-dimensional geometrical conformation provides a theoretically better environment for bone healing, increased anteroposterior and rotational stability, and safer opening-wedge loading force application with a low lateral hinge fracture risk. To better assess the advantages of this Z-shaped biplanar MOWHTO, in terms of osteotomy healing time, further studies, with higher level of evidence, are needed.

Furthermore, although the TKA conversion rate after Z-shaped MOWHTO is low, as emerged from this case series, studies with longer follow-up are required to further evaluate this novel technique.

\section{Abbreviations}

OA: Osteoarthritis; TKA: Total knee arthroplasty; HTO: High tibial osteotomy; LCWHTO: Lateral closing-wedge high tibial osteotomy; MOWHTO: Medial opening-wedge high tibial osteotomy; BMI: Body mass index; CPM: Continuous passive motion; VAS: Visual analogue scale; WOMAC: Western Ontario and McMaster University Osteoarthritis Index; TS: Tibial slope; CD: Caton-Deschamps index; MPTA: Medial proximal tibial angle.
Acknowledgements

The authors thank Prof. Raffale Garafolo, Dott. Emilio Romanini, and Dott. Alessandro Paglia for their help in the development of this project.

\section{Authors' contributions}

M.P. performed all the surgeries and is the major contributor in development of the surgical technique; R.G. was responsible for the concept of study, the assembly, analysis and interpretation of the data, and the drafting of the article; P.P. collected and analyzed the datasets of the study; M.G.M., A.G., F.R., P.P., and A.F. contributed to the writing of the article and interpretation of results M.A. was responsible for data and pictures collection; V.C. was the independent observer who performed the clinical and radiological assessment. All authors read and approved the final manuscript.

\section{Authors' information}

We want to dedicate this paper in honor of Dr. Marcello Presutti, the senior surgeon and close friend, who designed and developed the surgical technique of the opening-wedge high tibial Z-shaped osteotomy and performed all the surgeries of the study, a great and unforgotten physician who dedicated his life to the orthopedic art with an abiding interest in knee pathology and who prematurely passed away in May 2020.

\section{Funding}

The co-first author Remo Goderecci received a SIOT scholarship to perform this research (BORSA DI STUDIO SIOT 2019).

\section{Availability of data and materials}

The datasets used and/or analyzed during the current study are available from the corresponding author on reasonable request.

\section{Declarations}

\section{Ethics approval and consent to participate}

All procedures were performed in accordance with ethical standards, and the study protocol was approved by the Internal Review Board of our institution (authorization number 55/2019, 20 November 2019). Individual informed consent to participate in the study was obtained from all participants. The study complied with current national and international laws and regulations governing the use of human subjects (Declaration of Helsinki and its later amendments).

\section{Consent for publication \\ Not applicable.}

\section{Competing interests}

The authors declare that they have no competing interests. All authors certify that they have no affiliations with or involvement in any organization or entity with any financial interest, or nonfinancial interest in the subject matter or materials discussed in this manuscript.

\section{Author details \\ ${ }^{1}$ Unit of Orthopaedic Surgery, "Casa Di Cura Villa Esther", Bojano (Cb), Italy. ${ }^{2}$ Unit of Orthopaedics and Traumatology, "G. Mazzini" Civil Hospital of Teramo, ASL 4 Teramo, Piazza Italia 1, 64100 Teramo, Italy. ${ }^{3}$ Unit of Orthopaedics and Traumatology, Azienda Ospedaliero Universitaria - Ospedali Riuniti, Fog- gia (FG), Italy. ${ }^{4}$ Department of Life, Health and Environmental Sciences, Unit of Orthopaedics and Traumatology, University of L'Aquila, Piazzale Salvatore Tommasi 1, Blocco 11, 67100 L'Aquila (AQ), Italy. ${ }^{5}$ Hip Department (CAD) Gaetano Pini - CTO Orthopedic Institute, University of Milan, Milan (MI), Italy.}

Received: 17 August 2021 Accepted: 3 December 2021

Published online: 14 December 2021

References

1. Bitton R (2009) The economic burden of osteoarthritis. Am J Manag Care 15:S230-S235

2. Mina C, Garrett WE, Pietrobon R, Glisson R, Higgins L (2008) High tibial osteotomy for unloading osteochondral defects in the medial 
compartment of the knee. Am J Sports Med 36:949-955. https://doi.org/ 10.1177/0363546508315471

3. Messner K, Maletius W (1996) The long-term prognosis for severe damage to weight-bearing cartilage in the knee: a 14-year clinical and radiographic follow-up in 28 young athletes. Acta Orthop Scand 67:165-168. https://doi.org/10.3109/17453679608994664

4. Han SB, In Y, Oh KJ, Song KY, Yun ST, Jang KM (2019) Complications associated with medial opening-wedge high tibial osteotomy using a locking plate: a multicenter study. J Arthroplasty 34:439-445. https://doi.org/10. 1016/j.arth.2018.11.009

5. Brouwer GM, Van Tol AW, Bergink AP, Belo JN, Bernsen RMD, Reijman M, Pols HAP, Bierma-Zeinstra SMA (2007) Association between valgus and varus alignment and the development and progression of radiographic osteoarthritis of the knee. Arthritis Rheum 56:1204-1211. https://doi.org/ 10.1002/art.22515

6. Singh JA (2011) Epidemiology of knee and hip arthroplasty: a systematic review. Open Orthopaedics J 5:80-85. https://doi.org/10.2174/18743 25001105010080

7. Jacobs CA, Christensen CP, Karthikeyan T (2016) An intact anterior cruciate ligament at the time of posterior cruciate ligament-retaining total knee arthroplasty was associated with reduced patient satisfaction and inferior pain and stair function. J Arthroplasty 31:1732-1735. https://doi. org/10.1016/j.arth.2016.01.026

8. Riddle DL, Perera RA, Jiranek WA, Dumenci L (2015) Using surgical appropriateness criteria to examine outcomes of total knee arthroplasty in a United States sample. Arthritis Care Res 67:349-357. https://doi.org/10. 1002/acr.22428

9. Mittal A, Meshram P, Kim WH, Kim TK (2020) Unicompartmental knee arthroplasty, an enigma, and the ten enigmas of medial UKA. J Orthopaedics Traumatol 21:15. https://doi.org/10.1186/s10195-020-00551-x

10. Jennings JM, Kleeman-Forsthuber LT, Bolognesi MP (2019) Medial unicompartmental arthroplasty of the knee. J Am Acad Orthop Surg 27:166-176. https://doi.org/10.5435/JAAOS-D-17-00690

11. Konopka JF, Gomoll AH, Thornhill TS, Katz JN, Losina E (2015) The costeffectiveness of surgical treatment of medial unicompartmental knee osteoarthritis in younger patients: a computer model-based evaluation. J Bone Joint Surg Am 97:807-817. https://doi.org/10.2106/JBJS.N.00925

12. Herman B, Giffin JR (2016) High tibial osteotomy in the ACL-deficient knee with medial compartment osteoarthritis. J Orthopaedics Traumatol 17:277-285. https://doi.org/10.1007/s10195-016-0413-z

13. He M, Zhong X, Li Z, Shen K, Zeng W (2021) Progress in the treatment of knee osteoarthritis with high tibial osteotomy: a systematic review. Syst Rev 10:56. https://doi.org/10.1186/s13643-021-01601-z

14. Liu X, Chen Z, Gao Y, Zhang J, Jin Z (2019) high tibial osteotomy: review of techniques and biomechanics. J Healthcare Eng 2019:8363128. https:// doi.org/10.1155/2019/8363128

15. Marcheggiani Muccioli GM, Fratini S, Cammisa E, Vaccari V, Grassi A, Bragonzoni L, Zaffagnini S (2019) Lateral closing wedge high tibial osteotomy for medial compartment arthrosis or overload. Clin Sports Med 38:375-386. https://doi.org/10.1016/j.csm.2019.02.002

16. Woodacre T, Ricketts M, Evans JT, Pavlou G, Schranz P, Hockings M, Toms A (2016) Complications associated with opening wedge high tibial osteotomy - a review of the literature and of 15 years of experience. Knee 23:276-282. https://doi.org/10.1016/j.knee.2015.09.018

17. Liu JN, Agarwalla A, Gomoll AH (2019) High tibial osteotomy and medial meniscus transplant. Clin Sports Med 38:401-416. https://doi.org/10. 1016/j.csm.2019.02.006

18. Dubrana F, Lecerf G, Nguyen-Khanh JP, Menard R, Ardouin L, Gibon Y, Pidhorz L, Falaise V, Coipeau P, Burdin P, Rouvillain JL, Navarre T, Garron E, Daoud W, Louboutin H, Moineau G, Wessely L, Stindel E, Debarge R, Lustig S, Lavoie F, Neyret P (2008) Ostéotomie tibiale de valgisation. Rev Chir Orthop Reparatrice Appar Mot 94:2-21. https://doi.org/10.1016/j.rco. 2008.03.006

19. Brouwer RW, Huizinga MR, Duivenvoorden T, van Raaij TM, Verhagen AP, Bierma-Zeinstra SMA, Verhaar JA (2014) Osteotomy for treating knee osteoarthritis. Cochrane Database System Rev. https://doi.org/10.1002/ 14651858.CD004019.pub4

20. Han JH, Yang J-H, Bhandare NN, Suh DW, Lee JS, Chang YS, Yeom JW, Nha KW (2016) Total knee arthroplasty after failed high tibial osteotomy: a systematic review of open versus closed wedge osteotomy. Knee
Surg Sports Traumatol Arthrosc 24:2567-2577. https://doi.org/10.1007/ s00167-015-3807-1

21. Naudie DDR, Amendola A, Fowler PJ (2004) Opening wedge high tibial osteotomy for symptomatic hyperextension-varus thrust. Am J Sports Med 32:60-70. https://doi.org/10.1177/0363546503258907

22. Ogawa $\mathrm{H}$, Matsumoto K, Akiyama $\mathrm{H}$ (2017) The prevention of a lateral hinge fracture as a complication of a medial opening wedge high tibial osteotomy. Bone Joint J 99B:887-893. https://doi.org/10.1302/0301-620X. 99B7.BJJ-2016-0927.R1

23. Hankemeier S, Mommsen P, Krettek C, Jagodzinski M, Brand J, Meyer C, Meller R (2010) Accuracy of high tibial osteotomy: comparison between open- and closed-wedge technique. Knee Surg Sports Traumatol Arthrosc 18:1328-1333. https://doi.org/10.1007/s00167-009-1020-9

24. Hernigou P, Medevielle D, Debeyre J, Goutallier D (1987) Proximal tibial osteotomy for osteoarthritis with varus deformity. a ten to thirteen-year follow-up study. J Bone Joint Surg Am 69:332-354

25. Koshino T, Murase T, Saito T (2003) Medial opening-wedge high tibial osteotomy with use of porous hydroxyapatite to treat medial compartment osteoarthritis of the knee. J Bone Joint Surg Ser A 85:78-85. https:// doi.org/10.2106/00004623-200301000-00013

26. Lobenhoffer P, Agneskirchner JD (2003) Improvements in surgical technique of valgus high tibial osteotomy. Knee Surg Sports Traumatol Arthrosc 11:132-138. https://doi.org/10.1007/s00167-002-0334-7

27. Lobenhoffer P, Simoni C, Staubli A (2002) Open-wedge high-tibial osteotomy with rigid plate fixation. Techn Knee Surg 1:93-105. https://doi. org/10.1097/00132588-200212000-00004

28. Gaasbeek RDA, Sonneveld H, van Heerwaarden RJ, Jacobs WCH, Wymenga AB (2004) Distal tuberosity osteotomy in open wedge high tibial osteotomy can prevent patella infera: a new technique. Knee 11:457-461. https://doi.org/10.1016/j.knee.2004.02.002

29. van Heerwaarden $R$, Najfeld M, Brinkman M, Seil R, Madry H, Pape D (2013) Wedge volume and osteotomy surface depend on surgical technique for distal femoral osteotomy. Knee Surg Sports Traumatol Arthrosc 21:206-212

30. Rosenberg TD, Paulos LE, Parker RD, Coward DB, Scott SM (1988) The forty-five-degree posteroanterior flexion weight-bearing radiograph of the knee. J Bone Joint Surg Am 70:1479-1483

31. Ahlbäck S (1968) Osteoarthrosis of the knee. a radiographic investigation. Acta Radiol Diagn 277:7-72

32. Gould D, Kelly D, Goldstone L, Gammon J (2001) Examining the validity of pressure ulcer risk assessment scales: developing and using illustrated patient simulations to collect the data. J Clin Nurs 10:697-706. https:// doi.org/10.1046/j.1365-2702.2001.00525.x

33. Bellamy N, Buchanan WW, Goldsmith CH, Campbell J, Stitt LW (1988) Validation study of WOMAC: a health status instrument for measuring clinically important patient relevant outcomes to antirheumatic drug therapy in patients with osteoarthritis of the hip or knee. J Rheumatol 15:1833-1840

34. Likert R (1932) A technique for the measurement of attitudes. Arch Psychol 22(140):55

35. Brazier J, Migaud H, Gougeon F, Cotten A, Fontaine C, Duquennoy A (1996) Evaluation of methods for radiographic measurement of the tibial slope. A study of 83 healthy knees. Rev Chir Orthop Reparatrice Appar Mot 82:195-200

36. Caton J, Deschamps G, Chambat P, Lerat JL, Dejour H (1982) Patella infera. Apropos of 128 cases. Rev Chir Orthop Reparatrice Appar Mot 68:317-325

37. Kubota M, Ohno R, Sato T, Yamaguchi J, Kaneko H, Kaneko K, Ishijima M (2019) The medial proximal tibial angle accurately corrects the limb alignment in open-wedge high tibial osteotomy. Knee Surg Sports Traumatol Arthrosc 27:2410-2416. https://doi.org/10.1007/s00167-018-5216-8

38. El-Azab H, Halawa A, Anetzberger H, Imhoff AB, Hinterwimmer S (2008) The effect of closed- and open-wedge high tibial osteotomy on tibial slope: a retrospective radiological review of 120 cases. J Bone Joint Surg Ser B 90:1 193-1197. https://doi.org/10.1302/0301-620X.90B9.20688

39. Goshima K, Sawaguchi T, Shigemoto K, I wai S, Fujita K, Yamamuro Y (2019) Comparison of clinical and radiologic outcomes between normal and overcorrected medial proximal tibial angle groups after open-wedge high tibial osteotomy. Arthroscopy 35:2898-2908.e1. https://doi.org/10. 1016/j.arthro.2019.04.030 
40. Lee YS, Kang JY, Lee MC, On WS, Elazab A, Song MK (2016) Effect of the osteotomy length on the change of the posterior tibial slope with a simple distraction of the posterior gap in the uni- and biplanar open-wedge high tibial osteotomy. Arthroscopy 32:263-271. https://doi.org/10.1016/j. arthro.2015.07.018

41. Brinkman JM, Lobenhoffer P, Agneskirchner JD, Staubli AE, Wymenga $A B$, Van Heerwaarden RJ (2008) Osteotomies around the knee: patient selection, stability of fixation and bone healing in high tibial osteotomies. J Bone Joint Surg Ser B 90:1548-1557

42. Türkmen F, Kaçıra BK, Özkaya M, Erkoçak ÖF, Acar MA, Özer M, Toker S, Demir T (2017) Comparison of monoplanar versus biplanar medial opening-wedge high tibial osteotomy techniques for preventing lateral cortex fracture. Knee Surg Sports Traumatol Arthrosc 25:2914-2920. https://doi.org/10.1007/s00167-016-4049-6

43. Takeuchi R, Ishikawa H, Kumagai K, Yamaguchi Y, Chiba N, Akamatsu Y, Saito T (2012) Fractures around the lateral cortical hinge after a medial opening-wedge high tibial osteotomy: a new classification of lateral hinge fracture. Arthroscopy 28:85-94. https://doi.org/10.1016/j.arthro 2011.06.034

44. Rose T, Imhoff AB (2007) Complications after transgenicular osteotomies. Oper Tech Orthop 17:80-86. https://doi.org/10.1053/j.oto.2006.09.014

45. Coventry MB, Ilstrup DM, Wallrichs SL (1993) Proximal tibial osteotomy. A critical long-term study of eighty-seven cases. J Bone Joint Surg Am 75:196-201. https://doi.org/10.2106/00004623-199302000-00006

46. Pape D, Adam F, Rupp S, Seil R, Kohn D (2004) Stability, bone healing and loss of correction after valgus realignment of the tibial head. A roentgen stereometry analysis. Der Orthopade 33:208-217. https://doi.org/10. 1007/s00132-003-0591-2

47. Pape D, Dueck K, Haag M, Lorbach O, Seil R, Madry H (2013) Wedge volume and osteotomy surface depend on surgical technique for high tibial osteotomy. Knee Surg Sports Traumatol Arthrosc 21:127-133. https://doi. org/10.1007/s00167-012-1913-x

48. Staubli AE (2008) Radiological examination of bone healing after openwedge tibial osteotomy. In: Lobenhoffer PP, van Heerwaarden RJ, Staubli AE et al (eds) Osteotomies around the Knee, 1st edn. Thieme AO Foundation, Stuttgart, pp 133-146. https://doi.org/10.1055/b-0034-9892

49. Lind-Hansen TB, Lind MC, Nielsen PT, Laursen MB (2016) Openwedge high tibial osteotomy: RCT 2 years RSA follow-up. J Knee Surg 29(08):664-672
50. Goshima K, Sawaguchi T, Shigemoto K, Iwai S, Nakanishi A, Inoue D, Shima $Y$ (2019) Large opening gaps, unstable hinge fractures, and osteotomy line below the safe zone cause delayed bone healing after open-wedge high tibial osteotomy. Knee Surg Sports Traumatol Arthrosc 27(4):1291-1298. https://doi.org/10.1007/s00167-018-5334-3 (Epub 2018 Dec 11)

51. Staubli AE, Jacob HA (2010) Evolution of open-wedge high-tibial osteotomy: experience with a special angular stable device for internal fixation without interposition material. Int Orthop 34(2):167-172. https:// doi.org/10.1007/s00264-009-0902-2

52. Pape D, Lorbach O, Schmitz C, Busch LC, van Giffen N, Seil R, Kohn DM (2010) Effect of a biplanar osteotomy on primary stability following high tibial osteotomy: a biomechanical cadaver study. Knee Surg Sports Traumatol Arthrosc 18:204-211. https://doi.org/10.1007/s00167-009-0929-3

53. Belsey J, Yasen SK, Jobson S, Faulkner J, Wilson AJ (2021) Return to physical activity after high tibial osteotomy or unicompartmental knee arthroplasty: a systematic review and pooling data analysis. Am J Sports Med 49(5):1372-1380. https://doi.org/10.1177/0363546520948861

54. Niinimäki TT, Eskelinen A, Mann BS, Junnila M, Ohtonen P, Leppilahti $J$ (2012) Survivorship of high tibial osteotomy in the treatment of osteoarthritis of the knee: Finnish registry-based study of 3195 knees. J Bone Joint Surg 94(11):1517-1521

55. Spahn G, Hofmann GO, von Engelhardt LV, Li M, Neubauer H, Klinger HM (2013) The impact of a high tibial valgus osteotomy and unicondylar medial arthroplasty on the treatment for knee osteoarthritis: a metaanalysis. Knee Surg Sports Traumatol Arthrosc 21(1):96-112. https://doi. org/10.1007/s00167-011-1751-2

56. Robertsson O,W-Dahl A (2015) The risk of revision after TKA is affected by previous HTO or UKA. Clin Orthop Relat Res 473(1):90-93. https://doi.org/ 10.1007/s11999-014-3712-9

57. Schallberger A, Jacobi M, Wahl P, Maestretti G, Jakob RP (2011) High tibial valgus osteotomy in unicompartmental medial osteoarthritis of the knee: a retrospective follow-up study over 13-21 years. Knee Surg Sports Traumatol Arthrosc 19:122-127. https://doi.org/10.1007/s00167-010-1256-4

\section{Publisher's Note}

Springer Nature remains neutral with regard to jurisdictional claims in published maps and institutional affiliations.

\section{Submit your manuscript to a SpringerOpen ${ }^{\circ}$ journal and benefit from:}

- Convenient online submission

- Rigorous peer review

- Open access: articles freely available online

- High visibility within the field

- Retaining the copyright to your article

Submit your next manuscript at $\boldsymbol{\nabla}$ springeropen.com 